\title{
HispanismeS
}

Revue de la Société des Hispanistes Français

$18 \mid 2021$

Murs, barrières, obstacles dans les mondes hispaniques II

\section{El arte de volar d'Antonio Altarriba (2009)}

une dialectique entre les murs et le vol

Antonio Altarriba's El arte de volar (2009) : a dialectic between walls and flight El arte de volar de Antonio Altarriba (2009): una dialéctica entre los muros y el vuelo

\section{Emmanuelle Souvignet}

\section{OpenEdition}

Journals

Édition électronique

URL : https://journals.openedition.org/hispanismes/14170

DOI : 10.4000/hispanismes. 14170

ISSN : 2270-0765

Éditeur

Société des Hispanistes Français

Référence électronique

Emmanuelle Souvignet, «El arte de volar d'Antonio Altarriba (2009) », HispanismeS [En ligne], 18| 2021, mis en ligne le 31 décembre 2021, consulté le 30 janvier 2022. URL : http://journals.openedition.org/ hispanismes/14170 ; DOI : https://doi.org/10.4000/hispanismes.14170

Ce document a été généré automatiquement le 30 janvier 2022.

\section{c) (†) $\odot$}

Les contenus de cette revue sont mis à disposition selon les termes de la Licence Creative Commons Attribution - Pas d'Utilisation Commerciale - Pas de Modification 4.0 International. 


\title{
El arte de volar d'Antonio Altarriba (2009)
}

\author{
une dialectique entre les murs et le vol \\ Antonio Altarriba's El arte de volar (2009) : a dialectic between walls and flight \\ El arte de volar de Antonio Altarriba (2009): una dialéctica entre los muros y el \\ vuelo
}

\section{Emmanuelle Souvignet}

1 En 2009, Antonio Altarriba, en collaboration avec le dessinateur Kim, publie un roman graphique, intitulé El arte de volar ${ }^{1}$, dans lequel il rend hommage à son père, qui porte le même nom que lui et qui est décédé en 2001. L'auteur y raconte le parcours individuel paternel en l'inscrivant dans l'histoire espagnole $\mathrm{du} \mathrm{xx}^{\mathrm{e}}$ siècle, depuis sa jeunesse dans les années 1910 jusqu'à son suicide en 2001, en passant par son engagement anarchiste au moment de la Guerre civile puis son retour dans l'Espagne franquiste après une période d'exil en France. La trajectoire paternelle s'achève dans une maison de retraite proche de Logroño, où le vieil homme finit par se donner la mort après plusieurs années de solitude et de dépression.

2 Tout au long de ce récit biographique, les murs et les obstacles occupent une place prépondérante, aussi bien dans les mots employés par l'auteur que dans les représentations graphiques proposées par Kim. Gilbert Durand rappelle que le mur marque symboliquement « une intention de séparation, de promotion du discontinu $»^{2}$. À ce titre, et en fonction de la perspective adoptée, il peut protéger contre une agression mais aussi isoler ou stopper une progression. Dans El arte de volar, point de protection : Antonio est sans cesse confronté à des murs qui freinent son cheminement personnel et empêchent souvent sa construction identitaire. Pourtant, le titre du roman graphique fait du motif du vol l'élément central de l'œuvre, utilisant ainsi une forme de légèreté pour ouvrir un récit somme toute assez sombre. C'est cette contradiction apparente entre les murs, symbole d'enfermement, et le vol, symbole d'ouverture, qui sera au centre de notre réflexion. En quoi la dialectique entre l'obstacle et l'envol prend-elle une signification à la fois individuelle et collective au 
sein du roman graphique? Comment le mur devient-il le symbole d'une frustration? Comment ces motifs permettent-ils d'exprimer le choc entre un idéal et une réalité ? Pour répondre à ces questions, nous donnerons tout d'abord du sens à la multiplication des obstacles dans le roman. Puis nous aborderons la question du vol, pour montrer qu'il n'a de sens que par la présence des murs. Cependant, si passer les murs suppose de voler, le vol est aussi à maintes reprises synonyme de chute. Dans un dernier temps de notre analyse, nous nous intéresserons donc au motif de la chute, peut-être nécessaire à l'envol définitif, nécessaire en tout cas à la construction identitaire du personnage.

\section{La poétique de l'obstacle}

3 La première vignette du roman graphique est une image de barrière, à savoir l'image d'une fenêtre fermée, entravée par des barreaux (p.11). Il s'agit de la fenêtre de l'infirmerie dans laquelle se trouve Antonio, le père de l'auteur, à la fin de sa vie. Le bâtiment est lui-même totalement quadrillé, tant au niveau du carrelage au sol que de la représentation très rectiligne des couloirs, jalonnés de portes, à l'image des cellules d'une prison (p. 11). Le roman est donc placé sous le signe de l'enfermement. À partir de ce point de départ temporel qui correspond au jour du suicide, le roman restitue, par une longue analepse, le récit de la vie du personnage. Force est alors de constater que chaque étape de la trajectoire vitale d'Antonio est marquée par la confrontation à des obstacles. Les murs et autres barrières, très concrètes ou plus symboliques, qui jalonnent son chemin sont autant de freins à sa construction identitaire et à son épanouissement personnel.

4 La première partie du récit («El coche de madera ») relate l'enfance d'Antonio, dans son village natal de Peñaflor. Les difficultés économiques poussent chaque famille d'agriculteurs à ériger des murs de pierres pour délimiter son terrain et éviter de cette façon les abus entre voisins. C'est ainsi que les murs finissent par envahir la campagne, par la morceler, au nom de la protection de la propriété. Au niveau graphique, les murs occupent alors une grande partie de la page, sont mis en exergue par l'utilisation de vues panoramiques et le narrateur ne peut que constater la transformation : «Peñaflor entró en la lista pronto innumerable de los pueblos que ponen barreras al campo » (p. 22). Ces murs privent Antonio et ses amis d'horizon. Ils se retrouvent bloqués derrière les murs, « sin vistas ». 




(C) 2020, Antonio Altarriba y Kim et (c) 2020, Norma Editorial por la edición en castellano

5 Les murs bouchent toute perspective d'avenir et Antonio ne peut que subir cet enfermement dans la misère de sa condition sociale : "Así que crecí con el horizonte cegado por una barrera de ambición... o, quizá más bien, de miseria.» (p. 23). De façon symbolique, le champ de blé, qui apparaît dans la vignette citée, érige alors un autre mur derrière lequel Antonio est condamné à un travail qui ne lui convient pas. Le mur représente donc, dans cette première étape de la vie d'Antonio, une barrière sociale qui enferme dans un modèle familial préétabli.

Dans la deuxième partie du récit («Las alpargatas de Durruti »), le narrateur retrace l'engagement politique d'Antonio depuis l'avènement de la Seconde République jusqu'à son retour d'exil en 1949. Les étapes majeures de la trajectoire d'Antonio à ce momentlà de sa vie sont, une nouvelle fois, associées à des murs ou des obstacles qui viennent entraver son cheminement personnel. Ainsi, quelques jours après sa mobilisation dans les forces franquistes, Antonio décide de franchir de nuit la ligne de front pour rejoindre le camp républicain. Il se trouve alors confronté à une double barrière, composée à la fois de barbelés et d'un ravin. Il doit donc ramper puis escalader pour atteindre son objectif (p. 56). La représentation graphique du passage du ravin joue sur un écho visuel et textuel entre la descente («descender») et la remontée («el ascenso ») pour symboliser la notion de franchissement. Dans ce cas-là, il s'agit d'un franchissement volontaire et réussi quoique difficile mais, après la victoire franquiste, Antonio se voit contraint de quitter l'Espagne et prend donc le chemin de l'exil. Il passe la frontière, autre barrière invisible, et se retrouve enfermé dans le camp français de Saint-Cyprien. Pas de murs en pierres sur cette plage : « era todo lo que nos ofrecían los franceses... arena, mar y cielo...» (p. 79), constate le narrateur. Ici, rien ne semble boucher l'horizon, tout est ouvert, mais la mer constitue pourtant une nouvelle forme 
de mur, un obstacle insurmontable. De l'autre côté, un grillage et des miradors empêchent le passage de la « raya » qui matérialise la frontière française, faisant bel et bien de la plage une véritable prison. Antonio parvient cependant à s'échapper du camp mais, dans une France sous occupation, les réfugiés espagnols républicains sont traqués. Antonio est ainsi une nouvelle fois arrêté et détourné de son chemin puisqu'il est enfermé dans une prison à ciel ouvert dans l'attente que quelqu'un décide de son destin. Dans cet épisode, les murs délimitent à nouveau l'espace des vignettes, bouchant totalement l'horizon (p. 101). Pourtant, le hasard veut que, cette fois, le mur se brise sous les bombes des Américains libérateurs.

7 Suite au décès de sa mère et face à son incapacité à trouver sa place dans un monde qui ne lui correspond pas, Antonio décide de rentrer en Espagne et de se soumettre au régime franquiste. Il se marie et trouve un emploi dans une entreprise de fabrication de galettes, dirigée par le mari de sa cousine, phalangiste opportuniste qui a su faire fortune grâce à des arrangements avec le pouvoir. Contrairement aux autres parties, pas de multiplication de murs occultants pour illustrer cette troisième étape de la vie du personnage («Galletas amargas»). Cependant, le dessinateur Kim offre ici un univers totalement clos. Il élimine presque systématiquement les espaces extérieurs. Toutes les scènes se situent à l'intérieur des maisons (entre les murs, donc) ou sont des scènes de rue qui n'ouvrent jamais sur le ciel. Le dessin est très souvent saturé, centré sur les personnages, comme pour signifier qu'Antonio est entré dans le moule franquiste et qu'il s'est résigné à ne plus voir l'horizon. Un épisode illustre parfaitement le moment où le protagoniste prend conscience de la nécessaire soumission silencieuse au régime franquiste. Antonio rencontre par hasard son ami républicain Lucio qui travaille dans les égouts et évoque avec lui les souvenirs du front. Lucio lui répond aussitôt : «¡Chssttt...! ¿Cómo se te ocurre hablar del frente...? Se nota que acabas de volver... ». Le narrateur fait alors le constat suivant :

Lucio no era el único que había cambiado de chaqueta... La mera supervivencia exigía la adhesión incondicional al régimen... No solo había que renunciar a los viejos ideales sino mostrar mayor fervor que los adeptos... Detrás de esos cambios había una tragedia personal tan profunda como inconfesable... No se trataba de traición sino de suicidio ideológico... Para afrontar el presente, debían acabar con el pasado... Morir para seguir vivos... (p. 139)

Retourner en Espagne constitue pour Antonio une véritable mort identitaire. Cette idée est résumée graphiquement par la descente de Lucio dans le tunnel souterrain des égouts, expression verticale d'un obstacle qui absorbe l'individu et l'empêche d'exister (p. 139). L'enfermement est ici implicite.

9 L'enfermement redevient explicite dans la dernière partie de la vie d'Antonio ( $\mathrm{La}$ madriguera del topo »), au moment de son entrée à la maison de retraite. L'horizon extérieur réapparaitt mais il n'est qu'une illusion inaccessible. Le début de la page 184 est à ce titre significatif. 
Fig. 2. L'élargissement du champ pour marquer l'enfermement (p. 184)
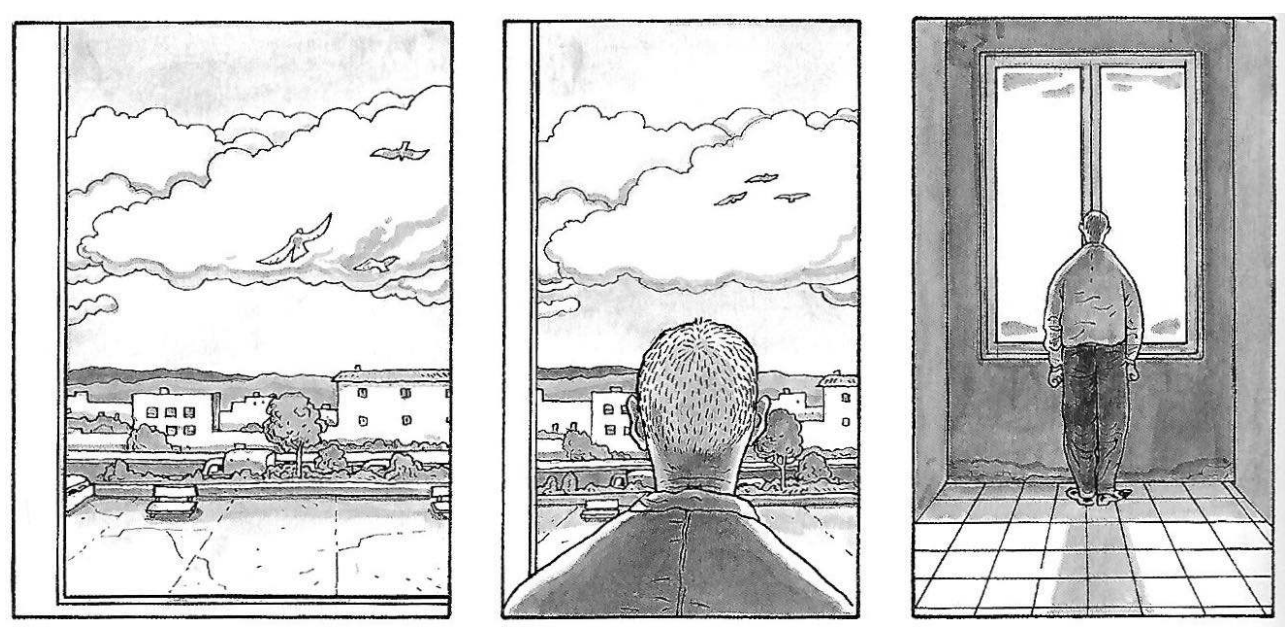

(C) 2020, Antonio Altarriba y Kim

(C) 2020, Norma Editorial por la edición en castellano

En effet, la première vignette offre un paysage ouvert, plein de quiétude avec ses arbres et ses oiseaux dans le ciel. Antonio apparaît de dos sur la deuxième vignette : il est comme inclus dans ce paysage. Mais, l'élargissement final du champ dans la troisième vignette fait disparaitre le paysage et révèle l'enfermement du personnage derrière une fenêtre de la maison de retraite. La barrière entre Antonio et le monde extérieur est ainsi clairement représentée : le personnage est dans l'incapacité d'avancer. Il ne peut que contempler l'extérieur sans y avoir accès. Cette technique est reprise quelques pages plus loin, lorsqu'Antonio est en train de se reposer dans la cour de l'établissement (p.192). On le voit tout d'abord sur son fauteuil, à travers un plan resserré. Puis, le plan s'élargit et le lecteur peut constater qu'il est en réalité face à un immense mur qui semble l'écraser. Antonio se retrouve littéralement «au pied du mur » : il n'a plus d'issue possible et ne peut qu'attendre impuissant la mort. Suite à ce constat, le personnage sombre d'ailleurs dans la dépression, incarnée par la taupe qui le ronge de l'intérieur, et qui a pour conséquence son enfermement définitif entre les murs de l'infirmerie.

11 Ce récapitulatif confirme que la trajectoire d'Antonio est sans cesse entravée. De façon récurrente, les murs bouchent l'horizon, laissant inaccessibles les rêves de changement. Dans El arte de volar, Antonio Altarriba utilise donc le motif du mur comme expression d'une frustration individuelle. Ces murs ou obstacles sont systématiquement associés à une réalité oppressante. Que ce soit l'autorité paternelle et la pression sociale pendant l'enfance, l'autorité des gendarmes français dans les camps et des soldats sous l'Occupation, l'autorité politique et corrompue du régime franquiste ou l'autorité médicale après l'installation à la maison de retraite, Antonio est sans cesse empêché par un environnement qui écrase l'individu et l'oblige à suivre une route toute tracée. Le motif de la route est d'ailleurs de nombreuses fois exploité par Kim mais souvent, au lieu d'ouvrir sur un possible, la route apparaît comme une voie à suivre, dont on ne peut pas dévier, comme si l'itinéraire de l'individu était prédéfini. Ainsi, la première partie du roman s'achève sur une image d'Antonio en train de quitter son village (p. 36) : il suit le chemin vers un monde plein d'espoir et pense ne jamais revoir son village de misère. Pourtant, au moment de sa mobilisation dans l'armée, il ne peut que revenir dans cet espace. La scène est alors presque identique mais la direction 
est opposée (p. 51). L'individu semble invariablement relié à ses origines. Mais, après avoir découvert la nouvelle situation de Peñaflor et avoir affronté sa désillusion, une vignette matérialise le départ définitif d'Antonio, toujours sur la même route, mais, cette fois-ci, le personnage est en train de vomir dans le fossé car la réalité est trop oppressante et écœurante (p.54). L'individu n'a pas le choix: il est poussé par le contexte à suivre telle ou telle route. De la même façon, au moment du passage de la frontière, la foule anonyme des émigrés suit la route. Chacun avance dans la même direction, sans savoir ce qui se trouve au bout du chemin. La route toute tracée est donc bien celle qui est imposée par la société ou par l'autorité (p. 78). À ce titre, la route peut être envisagée comme un nouvel obstacle dans la mesure où, loin d'ouvrir des perspectives, elle oriente en fait la trajectoire vitale de chacun.

Au-delà d'une frustration individuelle, le mur est aussi l'expression d'un empêchement collectif. Il symbolise tout ce qui écrase l'individu, freinant ainsi sa construction et son positionnement dans la société. Antonio Altarriba en fait une lecture clairement politique au début de la deuxième partie, lorsque le personnage d'Antonio arrive à Zaragosse, en pleine effervescence de l'avènement de la Seconde République. Il assiste à une manifestation dans laquelle sont exprimées les revendications sociales du peuple. Antonio se laisse emporter par l'enthousiasme général et lance un slogan significatif: « ¡Abajo los muros!» (p. 42).

Fig. 3. Abattre les murs (p. 42)

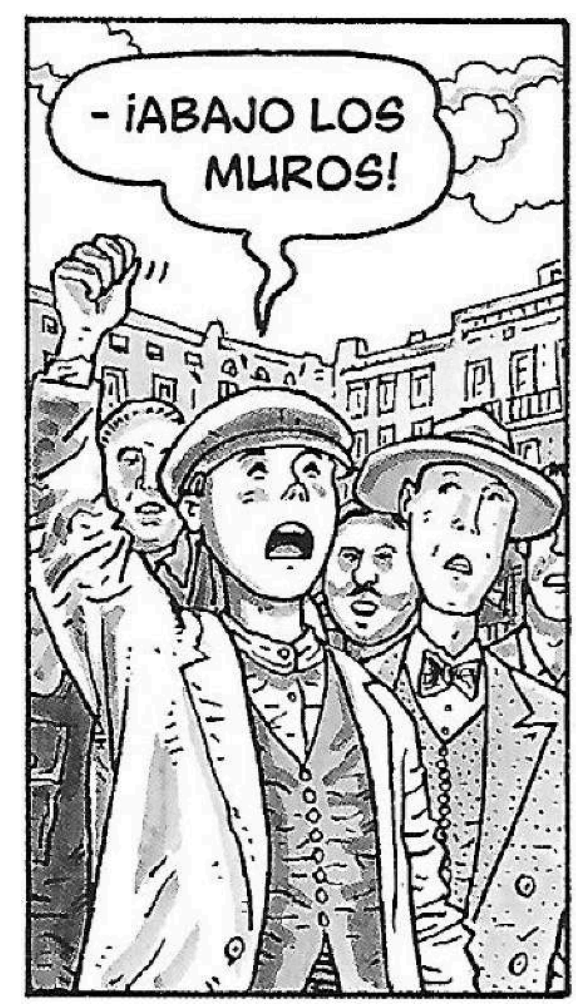

(C) 2020, Antonio Altarriba y Kim

(C) 2020, Norma Editorial por la edición en castellano

Les murs sont ici explicitement associés à la réalité collective oppressante qu'il faut abattre. Il est intéressant de remarquer que c'est justement cette image qui illustre la couverture des dernières éditions du roman graphique. Par ce choix, les éditeurs 
donnent, d'une part, une orientation de lecture politique, inscrivant la trajectoire du personnage dans l'histoire sociale $\mathrm{du} \mathrm{xx}^{\mathrm{e}}$ siècle, mais, d'autre part, ils placent indirectement le lien entre le vol et les murs au centre du roman graphique, en associant le verbe "volar» du titre à la réplique associée à l'image dans le texte, à savoir « abajo los muros». Par ce jeu paratextuel, la dialectique entre les murs et le vol semble bien être une clé de lecture de l'œuvre.

\section{Le vol ou la dynamique du dépassement}

14 Face à une réalité contraignante dont les murs sont infranchissables, le personnage d'Antonio ne peut se résoudre à rester au pied du mur. Tout au long de sa vie, il est ainsi guidé par l'obsession de voler car voler, c'est franchir les obstacles et pouvoir avancer. Si le motif des murs est, nous l'avons vu, omniprésent dans le roman, les allusions au vol le sont tout autant.

Lorsque les murs envahissent l'espace de leur enfance, Antonio et ses amis y voient un terrain de jeu. Ils montent sur les obstacles, comme pour les dominer malgré tout, y construisent des cabanes et s'amusent à sauter du haut des murs. Dans une forme d'insouciance, quand Antonio s'adonne à ce jeu, il a l'impression de voler : " ¡Es como volar...! » (p. 23), et de réaliser ainsi son rêve. Pourtant, nous verrons par la suite que ce vol est illusoire puisqu'il correspond en réalité à une chute. Au début de la Guerre civile, Antonio décide de rejoindre le camp républicain après sa mobilisation. Les vignettes qui illustrent le récit de sa réflexion font une nouvelle fois référence au vol. Antonio s'imagine en effet aux manettes d'un avion-machine à coudre, grâce auquel il peut bombarder les phalangistes qui l'avaient agressé dans la rue, en les cousant au sol. L'avion peut ensuite s'envoler dans les airs, au milieu des oiseaux, en un looping ascendant, symbole d'espoir (p. 51). De la même façon, les débuts de la vie amoureuse d'Antonio se passent dans les airs, lorsque le protagoniste emmène Petra faire un tour de grande roue. Pour répondre aux craintes de Petra, Antonio lui dit : «Además no hay que tener miedo a las alturas... Fíjate en los pájaros... La mejor manera de perder el vértigo es aprender a volar... Tranquila... » (p. 143). Au niveau graphique, la nacelle finit par se détacher de la structure métallique et entame un envol qui conduit les personnages dans les étoiles, le tout adossé au texte suivant :

Nunca había sentido nada igual por una mujer... Creo que, más que quererla, la necesitaba... Necesitaba la ternura de su mirada, la acogedora tibieza de sus brazos... Necesitaba, tras tanta intemperie y desventura, un lugar cálido donde acurrucarme. (p. 144)

L'association entre le texte et le dessin fait clairement du vol le moyen de surmonter les épreuves et de trouver un apaisement face aux « intempéries » de la réalité. Dans sa vie professionnelle, Antonio exprime également ses espoirs par l'image d'un vol onirique. Il s'imagine en train de survoler le monde, tel un super-héros juché sur une galette de l'entreprise dont il est devenu l'un des associés. Rien ne semble pouvoir l'arrêter.

17 Tout au long du roman graphique, un objet matérialise cette obsession de pouvoir voler : la voiture. Il donne d'ailleurs son titre à la première partie du roman, « El coche de madera ", comme pour faire de la voiture le point de départ de la trajectoire vitale d'Antonio. Pendant l'enfance, il s'agit tout d'abord de la voiture de Pepín, un fils de bonne famille du village. Antonio et son cousin Basilio observent avec envie les allées et venues de cet engin presque magique qui, sont-ils sûrs, peut voler (p. 27). La vignette 
qui reproduit la voiture de Pepín est immédiatement suivie d'une vignette centrée sur le ciel, au-dessus de la tête des enfants. L'association visuelle entre la voiture et l'espace $\mathrm{du}$ vol est ainsi explicite. Les deux amis ont d'ailleurs pour unique objectif de construire leur propre voiture pour pouvoir, eux aussi, voler au-dessus des murs et oublier ainsi l'enfermement de leur réalité :

Me gustaban los juegos que ayudaban a olvidar la realidad. Soñaba con ser conductor de automóviles y circular a toda velocidad por un mundo sin muros ni fronteras. Y así, sin más motor que la imaginación, me fugaba a kilómetros de distancia. (p. 29)

Le vol s'impose comme le contrepoint de la réalité et de ses murs. Il est assimilé au monde de l'imagination, du rêve, de l'évasion. La voiture symbolise alors le pont entre les deux univers. C'est pourquoi elle va jalonner la vie d'Antonio. Ainsi, dans sa vie politique et professionnelle, on attribue très souvent à Antonio la fonction de chauffeur. Il est d'abord chauffeur officiel de sa section de combattants anarchistes, chargé de livrer le courrier aux soldats. Et, comble du rêve, on le met au volant d'une « hispano-suiza ", semblable à celle de Pepín. Très vite, il décide de lui installer des ailes factices à l'arrière, pour symboliser son rôle de messager. Tel Hermès, il parcourt ainsi les zones en guerre et, malgré le danger, les moments passés au volant de son bolide sont sources d'un bonheur absolu. Les nombreuses vignettes qui illustrent cet épisode s'achèvent d'ailleurs sur une image de la voiture en plein vol, parmi les oiseaux (p. 66).

Fig. 4. La voiture comme symbole du vol (p. 66)

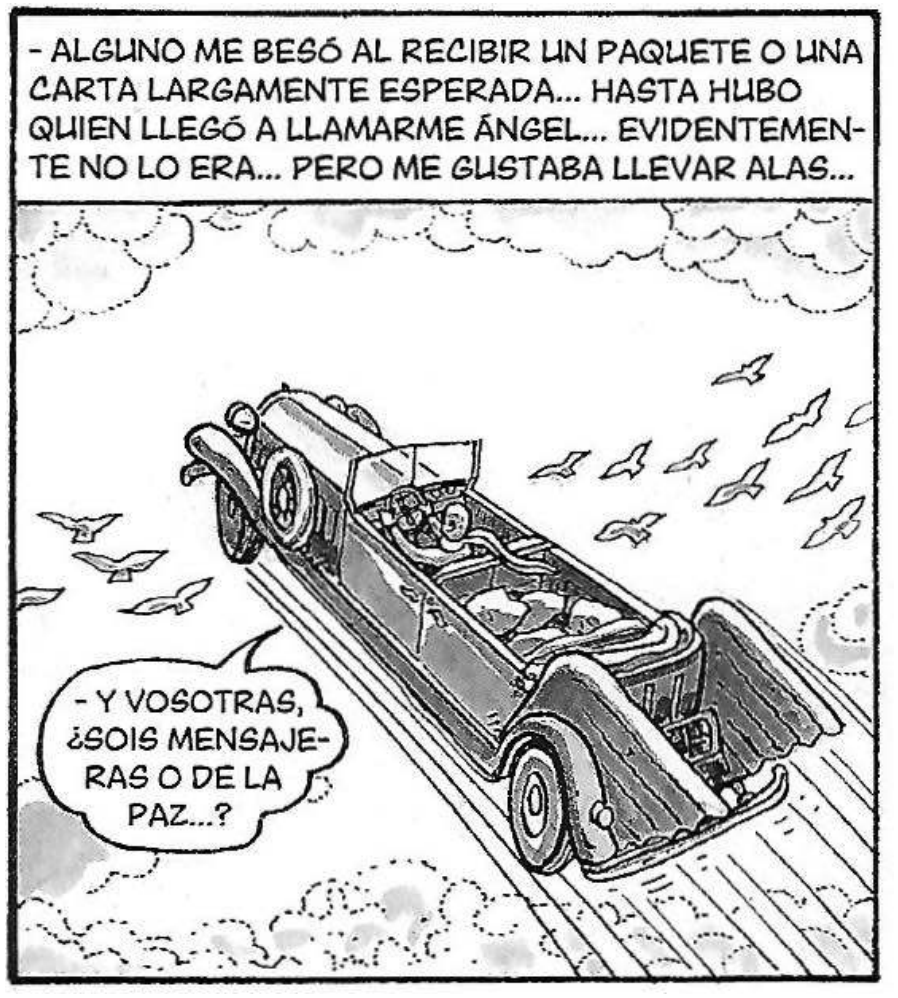

(c) 2020, Antonio Altarriba y Kim

(c) 2020, Norma Editorial por la edición en castellano 
19 Ensuite, lorsqu'il s'engage dans la Résistance française, il est une nouvelle fois embauché comme chauffeur. Son ami Pablo dit alors de lui : «Dejadle un coche y lo hará volar » (p. 108), associant là encore la voiture et le vol. Enfin, une fois rentré en Espagne, Antonio intègre l'entreprise de Doroteo en tant que chauffeur mais cet emploi symbolise alors l'obligation de participer, malgré lui, aux manœuvres de marché noir de ce dernier, ce qui ôte tout le plaisir de conduire au personnage. Finalement, si la voiture est sans cesse associée à l'obsession de voler, le récapitulatif qui vient d'être fait souligne cependant comment le symbole s'émousse peu à peu : il incarne d'abord le rêve fou d'un enfant plein d'espoir puis, face aux difficultés de la vie, il finit par devenir le simple instrument d'un monde corrompu. La quête de l'envol resurgit au cours de la dernière étape de la vie d'Antonio puisque, une fois en maison de retraite, il est embauché comme chauffeur par son ami Hipólito qui, depuis son fauteuil roulant, réclame quelqu'un pour le pousser. Le duo ainsi formé ne semble jamais aussi heureux que lorsqu'il dévale les pentes, à bord du nouveau bolide qu'est le fauteuil roulant aménagé (p. 189). Une nouvelle fois, l'engin est représenté en plein vol, au milieu du ciel.

Que ce soit une voiture, la nacelle d'une grande roue, un avion-machine à coudre ou un fauteuil roulant, nombreux sont les instruments qui incarnent le désir absolu de s'envoler dans El arte de volar. Leur point commun est qu'ils permettent de rêver, de tenter d'atteindre un idéal. Ils permettent de franchir les murs, ou de nier tout au moins leur existence. Ils permettent de toucher au bonheur en offrant un espace de liberté et d'espoir. Une vignette résume ce sentiment absolu d'ouverture vers un autre monde (p. 42). Antonio vient d'obtenir son permis de conduire et l'exhibe devant lui comme un véritable sésame, capable de faire effondrer les murs. 
Fig. 5. Le pouvoir du permis de conduire (p. 42)

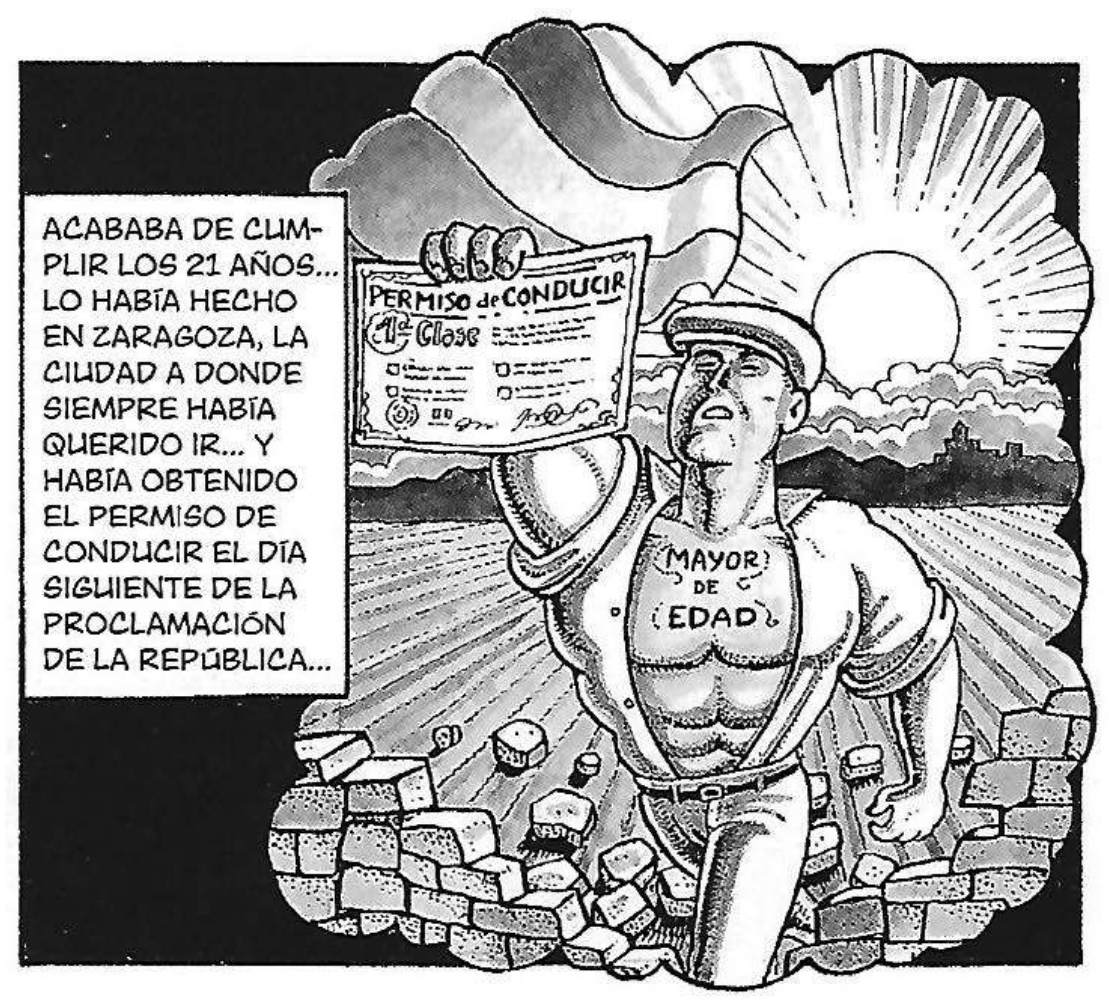

(c) 2020, Antonio Altarriba y Kim

(C) 2020, Norma Editorial por la edición en castellano

21 En une image unique, Kim réunit tous les espoirs du personnage à ce moment-là de sa vie : la majorité («mayor de edad»), c'est-à-dire la fin de l'autorité paternelle et la liberté de choisir ce que sera sa vie ; le permis de conduire, c'est-à-dire la possibilité d'avancer, d'aller vers autre chose, de voler; le drapeau de la République associé à un soleil qui se lève, comme signes de l'espoir en un nouvel ordre social et politique; le mur détruit par la marche déterminée d'un Antonio fort et invincible, comme pour montrer que rien n'est impossible désormais. Ces mouvements d'espoir, où le passage dans un autre monde idéal semble réalisable, sont nombreux dans le roman d'Altarriba, tout au moins au début. Graphiquement, ils sont symbolisés par les fenêtres, ce qui peut paraître contradictoire puisque, comme le souligne Rafaela Brumwell, grâce à la fenêtre, « on peut accéder à l'extérieur par les sens [...] mais elle ne constitue pas un lieu de passage et elle ne renvoie pas non plus à un déplacement corporel $\star^{3}$, contrairement à la porte. Or, la spécificité de El arte de volar est que, justement, le passage se fait par les fenêtres. Dans la première partie du roman, chaque fois qu'Antonio arrive à un moment-clé de son parcours, affirmant son envie de changer de vie, il franchit une fenêtre. Ainsi, lorsqu'il décide à deux reprises de quitter son village pour aller travailler à Zaragosse, il le fait de nuit en passant par la fenêtre. Au niveau de la construction visuelle, ces fuites par la fenêtre font contrepoint aux vignettes précédentes. En effet, dans le premier cas (p. 24), la fuite répond à une vignette sur laquelle Antonio avait dû faire face à l'autorité violente de son père. Dans le second cas (p. 36), elle répond à la tristesse et à la solitude d'Antonio à la suite du décès de son cousin Basilio. Après sa première tentative de fuite ratée, Antonio prend conscience de la nécessité d'apprendre pour pouvoir prendre son envol : 
con la edad experimenté una acuciante necesidad de aprender. Como ningún conocimiento podía servir en un pueblo donde bastaba con saber arar la tierra, deduje que todavía albergaba la esperanza de salir de allí. (p. 29) recherche de la hauteur et de l'ascension. Comme le rappelle Gaston Bachelard, toutes les opérations de l'esprit humain «n'ont qu'un axe de référence : l'axe vertical »" Contrairement au mouvement horizontal dont les murs empêchent la progression, "l'ascension n'a pas d'obstacles " ${ }^{6}$. Il s'agit donc, pour Antonio, de toujours monter pour voir au-delà des murs. Ainsi, pendant son enfance, Antonio a l'habitude de se hisser au sommet d'un noyer pour observer l'horizon et réfléchir à ses rêves et à son avenir : «-Y ahora, ¿cómo haremos para mirar a lo lejos ? - Podemos subirnos al nogal del perico. » (p. 23). Les vues panoramiques dessinées alors par Kim (p. 24, p. 27 et p. 36) sont le symbole d'une volonté d'envol.

Fig. 6. Le noyer ou la nécessaire ascension (p. 24)
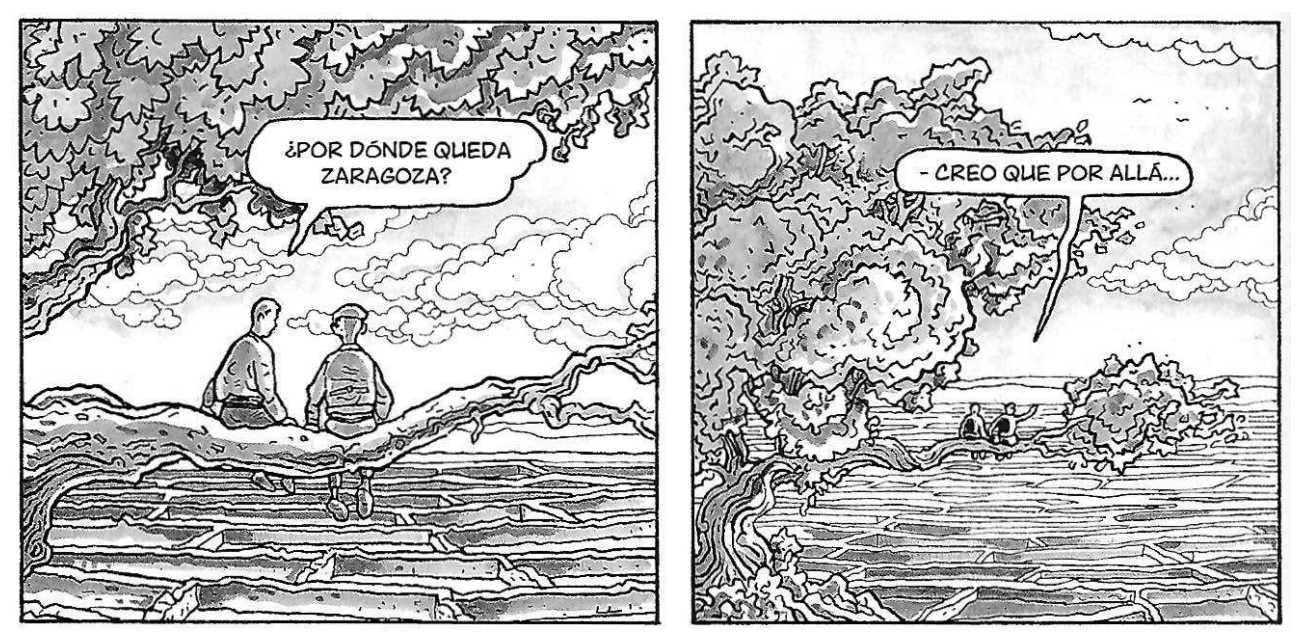

(c) 2020, Antonio Altarriba y Kim

(c) 2020, Norma Editorial por la edición en castellano

Le noyer devient l'observatoire du monde et l'espoir d'une vie meilleure est bien présent. Une fois dans sa vie d'adulte, comme nous l'avons déjà dit, l'horizon est davantage bouché pour Antonio et les occasions d'ascension sont beaucoup plus rares. Il est alors coincé dans une horizontalité qui l'oblige à faire face aux obstacles, à affronter la réalité. Le motif de l'ascension réapparaît dans la dernière étape de sa vie, au moment de son suicide, car « l'être qui monte voit s'effacer les dessins de l'abîme " ${ }^{7}$. À ce titre, la construction du prologue est significative. La fenêtre entravée de sa 
chambre constituant un obstacle à la défenestration, Antonio entame une ascension jusqu'au quatrième étage de la maison de retraite pour accéder à une fenêtre ouverte. Il monte tout d'abord les escaliers dont les marches sont autant d'épreuves physiques qu'il doit endurer pour atteindre la libération, comme une étape initiatique qu'il mènerait enfin à bien au crépuscule de sa vie (p. 12). Une fois arrivé en haut, il grimpe encore sur une chaise pour pouvoir accéder au rebord de la fenêtre. Kim propose alors une vue plongeante jusqu'en bas de l'immeuble, effet visuel qui accentue l'impression de hauteur (p.13). Les "dessins de l'abîme" reparaissent ici mais la position de domination est nécessaire pour l'envol final d'Antonio qui, de la sorte, semble reprendre son destin en main. Nous verrons plus loin que la défenestration d'Antonio est envisagée comme la réalisation du rêve de vol. Les pensées du personnage confirment d'ailleurs l'objectif : «y ahora a volar... » (p. 13). Du début à la fin de sa vie, et malgré les obstacles, Antonio n'aura été guidé que par l'idée de voler. Il n'aura jamais renoncé à son idéal, quitte à l'atteindre dans la mort.

Le roman graphique d'Antonio Altarriba est finalement l'expression de la lutte entre deux forces contraires : une force qui freine, qui empêche (les murs) et une force qui élève et qui est capable de nier les obstacles (le vol). Comme nous l'indique le titre, voler devient « un art » difficile, mais pourtant essentiel, car il permet de croire en ses rêves. L'histoire d'Antonio, c'est l'histoire d'une confrontation entre un idéal et la réalité, c'est l'histoire d'un homme qui se heurte parfois violemment aux barrières érigées par la réalité mais qui s'agrippe à son espoir pour pouvoir les franchir. Mais la lutte est sans merci et entraîne très souvent la chute. Le prologue résume à lui seul cette idée de chute: lorsqu'Antonio pense " $\mathrm{Y}$ ahora a volar », le narrateur précise : « mi padre tardó noventa años en caer de la cuarta planta » (p. 13). Le passage du verbe "volar» au verbe «caer» condense toute l'ambigüité du vol chez Antonio Altarriba, idée qui pourrait être résumée dans une question posée par Gaston Bachelard à propos du vol onirique: "Le vol onirique n'a-t-il pas pour fonction de nous apprendre à surmonter notre peur de tomber? $»^{8}$.

\section{Le motif de la chute : échec ou libération?}

Certes le rêve de vol est omniprésent dans le roman, comme unique moyen pour tenter de franchir les obstacles mis en travers de la trajectoire individuelle de chacun, comme unique moyen pour tenter de vaincre la gravité qui contraint l'être humain à rester au sol, au cœur des tourments. Mais ce rêve est systématiquement voué à l'échec. Ainsi, le revers négatif de l'envol est la chute, très souvent brutale, comme le soulignent Jean Chevalier et Alain Gheerbrant dans leur Dictionnaire des symboles, à propos du rêve de voler :

Dans les mythes et dans les rêves, le vol exprime un désir de sublimation, de recherche d'une harmonie intérieure [...]. Plus ce désir s'exalte, plus cette incapacité évolue en angoisse [...]. Le rêve d'envol se termine en cauchemar de chute : expression symbolique de la réalité vécue, des échecs réels9.

27 La «réalité vécue» est celle des murs, des obstacles qui conduisent donc inévitablement à la chute. Ainsi, si, pendant l'enfance, Antonio avait l'illusion de voler en sautant du haut des murs, au moment du récit biographique, le narrateur reconnait : " pero nunca crecí lo suficiente para superarlas definitivamente » (p. 23). Le roman est placé sous le signe de l'incapacité à dépasser les barrières. Nous avons montré 
précédemment comment la voiture symbolise le désir de s'envoler, de se libérer de ses chaînes, mais, dans la réalité, elle est généralement vouée à la destruction. Lorsqu'Antonio et Basilio font l'essai de la voiture qu'ils ont construite, ils perdent vite le contrôle et la voiture s'encastre dans un mur (p. 33). " ¡Putos muros de mierda...! », s'écrie alors le personnage, exclamation qui résume le drame du protagoniste qui, quoi qu'il fasse, finit dans les murs.

Fig. 7. L'échec du franchissement (p. 33)

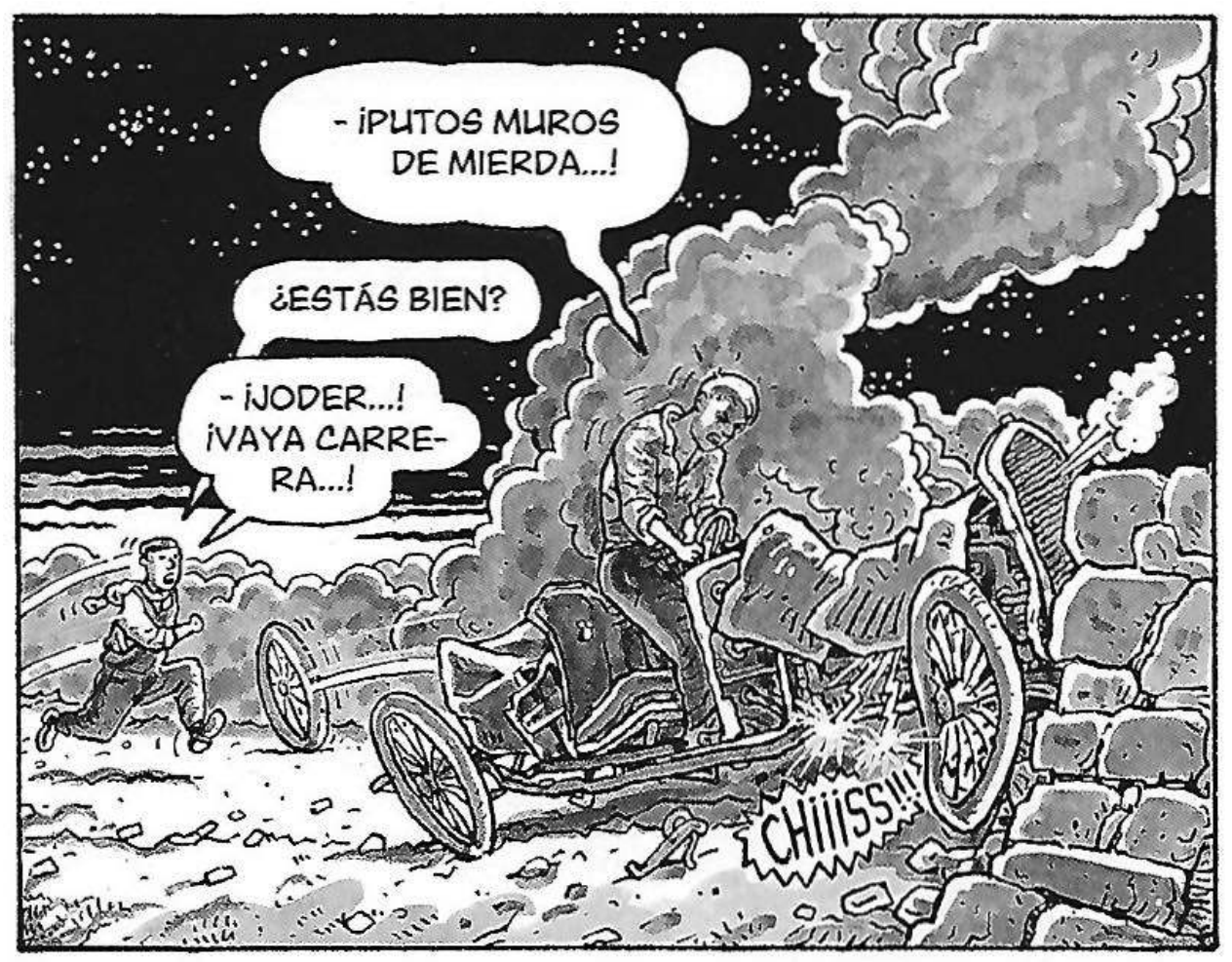

(c) 2020, Antonio Altarriba y Kim

(c) 2020, Norma Editorial por la edición en castellano

28 L'expression est répétée quelques pages plus loin, quand la course automobile s'achève, de façon plus dramatique cette fois, par la mort de Basilio. Antonio invective alors les murs qu'il doit franchir et qui, par la présence de morceaux de verre à leur sommet, l'empêchent de courir au secours de son cousin. Les images associées à l'accident de Basilio sont une représentation parfaite de la chute (p.34): une première vignette illustre la vitesse de la voiture, prête à s'envoler, tandis que le récit indique «Basilio llegó a volar... », mais la phrase se termine sur la vignette suivante par les mots « ... solo durante unos segundos ", lorsque la voiture fait une embardée et s'élève dans les airs pour retomber violemment au sol sur la dernière vignette. De même, au moment de la Guerre civile, Antonio se voit contraint d'abandonner son " hispano-suiza », suite à une réorganisation des troupes. Mais, plutôt que de la voir passer dans d'autres mains, il préfère y mettre le feu. Il s'agit là d'une destruction très symbolique car elle révèle que, même si les rêves peuvent être touchés du doigt, ils sont toujours rattrapés par la réalité. La défaite face aux forces de Franco marque le début d'un récit ponctué par la notion d'échec. Ainsi, au moment de franchir la frontière française, Antonio fait un bilan désastreux de sa vie : « ese día cumplía 29 años... Me sentía viejo y, por supuesto, 
derrotado... » (p. 78). Une fois dans le camp de Saint-Cyprien, il constate : «Nuestros sueños de libertad terminaban en una pesadilla de espino... Éramos como pájaros construyendo nuestra propia jaula... » (p. 80). La vie d'Antonio n'est alors faite que de départs, d'arrestations, de fuites. Il en vient à perdre totalement ses repères, à perdre même son identité, perte qui est symbolisée par les difficultés à trouver de nouveaux papiers d'identité en France. La conséquence de ce sentiment d'échec exacerbé et de cette désillusion absolue est la soumission finale à l'ordre établi, marquée par le retour en Espagne. Avant de quitter Marseille, il décide de brûler les espadrilles qui l'avaient suivi tout au long de son engagement politique, signe d'un renoncement à son monde idéal. Si l'image du ciel est convoquée dans ce moment de transition, elle sert à suivre la fumée produite par la combustion des chaussures, fumée qui s'élève dans les airs en passant par la fenêtre de l'appartement (p. 128). La fenêtre reste un espace d'ouverture mais, cette fois-ci, elle est l'endroit par lequel s'envolent tous les espoirs, laissant Antonio au sol.

Fig. 8. La fenêtre comme barrière pour l'individu (p. 128)
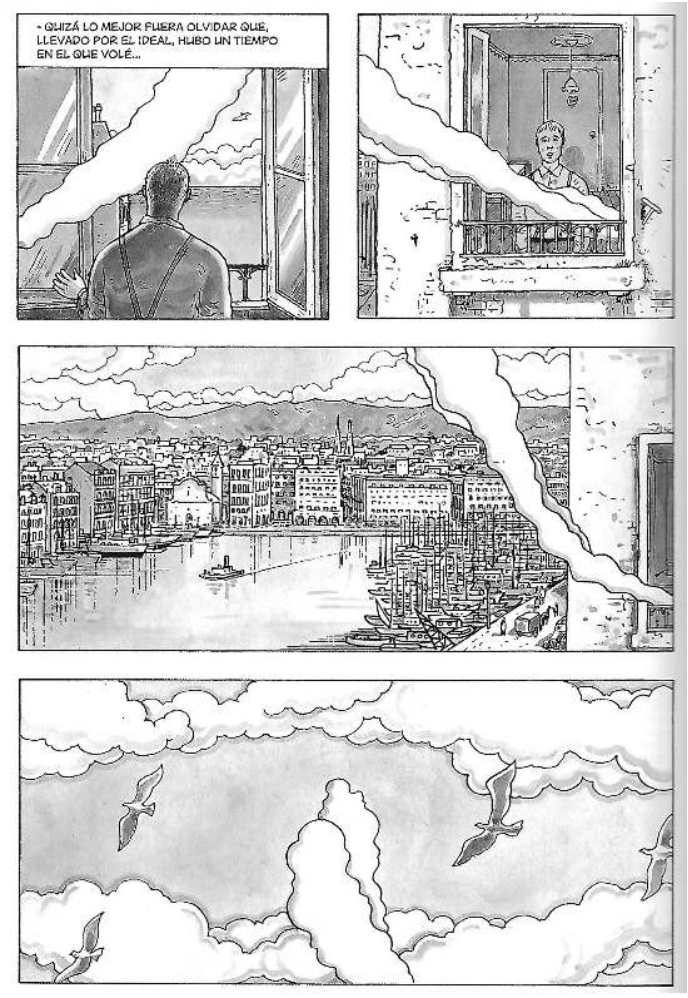

(C) 2020, Antonio Altarriba y Kim

(c) 2020, Norma Editorial por la edición en castellano

Il est d'ailleurs intéressant de constater que, dans la seconde moitié de sa vie, le personnage reste systématiquement derrière les fenêtres, contrairement aux fenêtres qu'il franchissait dans sa jeunesse. Ce traitement des fenêtres est le signe de l'échec du personnage. Il a perdu ses illusions de pouvoir atteindre l'inaccessible. Après avoir été ouverture, la fenêtre devient obstacle. Une fois en Espagne, nous avons vu que les références au vol disparaissent presque. Elles n'existent que dans l'imagination d'Antonio. Mais, même dans les rêves, ces scènes s'achèvent par une chute. Ainsi, lorsqu'Antonio s'invente en super-héros, chevauchant sa galette magique pour voler 
dans les airs, il ne maîtrise pas son atterrissage et chute au sol. De même, l'image de la grande roue, qui dans un premier temps s'envolait pour symboliser l'espoir en l'amour, est à nouveau convoquée à la fin de la partie dans un mouvement visuel inversé (p. 171). En effet, sur l'espace de quatre vignettes, Kim utilise la technique du zoom pour passer d'une grande roue qui semble encore voler à l'image d'une nacelle vide, tenant difficilement en équilibre sur un mécanisme grinçant et prêt, semble-t-il, à s'écrouler. De plus, cette nouvelle référence à la grande roue est visuellement reliée à l'alliance de plomb qu'Antonio décide de jeter, pour souligner l'abandon définitif de ses illusions. Rappelons qu'Antonio et ses amis avaient fondu ces alliances pendant la Guerre civile pour symboliser leur union dans la défense de leurs idéaux. Face au vide de son existence, le vol est dorénavant impossible et la chute semble inéluctable.

Entre les murs de la maison de retraite, face à un sentiment profond de solitude, Antonio trouve enfin l'issue en la chute ultime qu'est le suicide. Dans les derniers jours de sa vie, Antonio décide de reprendre sa vie en main. Cela commence par le meurtre symbolique de la taupe qui le ronge de l'intérieur. Après une scène violente pendant laquelle il tue l'animal à pleines dents, les vignettes nous montrent un Antonio totalement apaisé qui s'endort enfin sereinement alors que la cicatrice de son ventre se referme. Dans son sommeil, il se retrouve face à un tribunal de l'au-delà constitué de ses proches décédés. Le verdict est sans appel: «Este tribunal ha acordado por unanimidad liberarte de la vida [...] Ya eres libre... Bueno, pues eso... Que te puedes morir cuando quieras... Yo diría más... Debes morirte cuanto antes...» (p. 203). La décision est prise : la liberté et la paix retrouvées trouveront leur expression dans une mort volontaire et planifiée.

Intéressons-nous davantage à la relation contradictoire entre le suicide et le vol dans la construction de $E l$ arte de volar. La représentation du suicide est ce qui structure le roman graphique. En effet, chaque titre de partie est illustré par une page qui associe une image de la chute du personnage et l'étage auquel il se trouve dans une progression descendante cette fois, opposée à la logique ascensionnelle du vol. Le mur constitue ici la toile de fond de la chute comme pour signifier qu'Antonio a cessé de tenter de franchir les murs par le haut. Dorénavant, il les suit vers le bas, la dernière étape étant le choc avec le sol. Pourtant, les images de la chute fatale montrent un personnage qui semble voler dans les airs. Là est toute la contradiction du motif de la chute dans le roman. Le suicide illustre parfaitement ce que Bachelard appelle « la dialectique de la légèreté et de la lourdeur ${ }^{10}$, propre au rêve de vol. Antonio semble en effet totalement libre de ses mouvements, il arbore un visage épanoui et heureux (p. 37), il adopte même la position propre aux parachutistes en chute libre, avec les bras écartés pour profiter pleinement du moment, même si la veste qui vole au vent souligne la rapidité de la chute. 
Fig. 9. Le bonheur de la chute finale (p. 37)

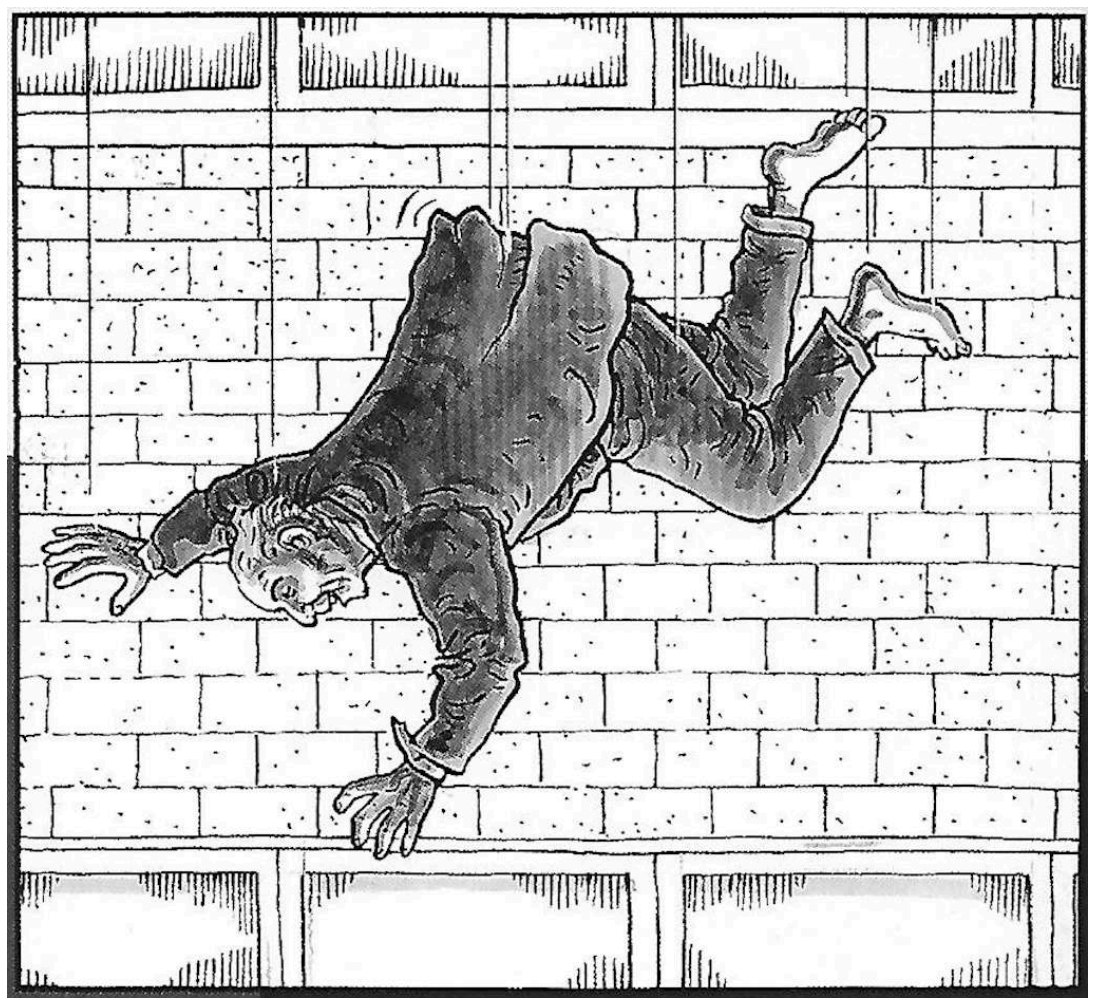

(c) 2020, Antonio Altarriba y Kim

(C) 2020, Norma Editorial por la edición en castellano

Cette chute effrénée le long du mur de la maison de retraite est le moment de plénitude qui lui permet de remonter le temps de la frustration, selon le schéma du vol inversé. « Ha llegado la hora... la hora de echar a volar» (p. 205) sont les derniers mots du roman qui sonnent comme un aboutissement. Au moment de mourir, Antonio réalise enfin son rêve, il a trouvé le moyen d'éviter le choc avec les murs, même si, là encore, tout cela n'est qu'une illusion comme l'affirme Álvaro Pons : « volar sin alas es sólo una ilusión que disfraza a la muerte $»^{11}$.

Pour trouver la résolution de la contradiction entre le vol et la chute et pour parvenir à franchir définitivement les murs, peut-être faut-il aller au-delà du récit et s'intéresser au projet biographique d'Antonio Altarriba fils, le créateur du roman graphique qui reconnaît que "la escritura en este caso surge de la impotencia. Como recurso desesperado, confesión o denuncia, cuando todo ha fallado $»^{12}$. En quoi l'œuvre littéraire peut-elle permettre à son père de prolonger son vol et de franchir ainsi toutes les barrières? L'œuvre d'Altarriba s'inscrit tout d'abord dans la thématique de la mémoire historique, très présente dans la société espagnole au début des années 2000, époque de gestation de $E l$ arte de volar. L'auteur veut rendre un vibrant hommage à son père et à tous ces Espagnols qui se sont battus pour défendre des idéaux, malgré les barrières de l'Espagne franquiste. Le motif du mur est exploité pour représenter la frustration individuelle de toute une génération mais aussi l'empêchement collectif dont elle a souffert et qui l'a empêchée de se construire une identité. Altarriba veut redonner cette identité digne et remarquable à ces hommes, à travers l'exemple de son père. Il les présente en ces termes: "Personas que vivieron una época histórica tremenda, que intentaron apostar por unos ideales y que nos han dejado un patrimonio 
de dignidad que no podemos marginar $»^{13}$. L'œuvre littéraire permet ainsi de laisser une trace qui survole les époques et donne de la hauteur aux protagonistes anonymes de l'histoire traumatique espagnole. Par cette trace qui dépasse les obstacles, Altarriba prend finalement en charge le vol empêché de son père comme pour répondre au souhait paternel, exprimé clairement dans le roman graphique, au moment de la naissance du fils : « No quiero que le pase como a mí... Quiero que aprenda a volar desde niño» (p. 148). Cette réplique répond aux inquiétudes de son épouse, lorsqu'Antonio joue à faire voler son fils dans les airs ( ¿Qué haces...? ¿No ves que el niño se te puede caer?»). Ce dialogue repose une nouvelle fois sur la dialectique entre la chute et le vol, entre la réalité et le rêve, la mère craignant la chute du bébé alors que le père veut l'inciter à se libérer. Se pose ici toute la question de la transmission entre générations. Le fils, c'est-à-dire l'auteur lui-même, doit pouvoir relayer les idéaux du père. C'est ce qu'il fait dans son récit graphique, grâce à un "proceso de transferencia $»^{14}$ qui lui permet d'incarner son propre père dans le récit et de raconter son histoire à la première personne. Le père devient ainsi un personnage de fiction dont l'art de voler peut passer à la postérité :

Pero en el momento en el que salta de la cuarta planta y emprende el vuelo, convertido en personaje de ficción y gracias al éxito del libro, llega a ser querido y comprendido por más gente de la que nunca se hubiera imaginado ${ }^{15}$.

L'épilogue du roman, ajouté en 2012 par l'auteur, confirme ce pouvoir de la création artistique. Altarriba s'inspire d'un échange réel avec une certaine Chantal, une lectrice rencontrée dans le sud de la France, lors d'une séance de dédicace, pour mettre le point final à son œuvre. Le témoignage de cette femme confirme à l'auteur qu'il a rempli sa mission puisqu'elle affirme que le vol existe :

He venido a decirle que no sufra por su padre... Tuvo una muerte feliz... Su cuerpo se llenó de luz y su caída se prolongó en el tiempo... Su padre realmente voló... Lo sé porque yo también me tiré por una ventana... [...] Mis motivos fueron distintos a los de su padre... Motivos de mujer... Pero también sufrí el autoritarismo y el agobio de los muros... [...] Cuando todas las puertas se cierran, está bien saber que siempre queda una ventana abierta... (p. 222)

Ces paroles résument à elles seules toute la problématique soulevée dans notre analyse. On y retrouve les murs qui étouffent, la dialectique entre les portes fermées et les fenêtres ouvertes, la tentative de vol ultime par le suicide et enfin l'idée de chute que celui-ci implique. Mais une chute qui «se prolong[e] dans le temps » n'est-elle pas un vol perpétuel ? En incarnant son père dans El arte de volar, Antonio Altarriba permet à ce dernier de voler hors de toute contingence temporelle; il lui donne une forme d'immortalité dans le vol. Grâce à l'écriture et aux nombreux lecteurs, le vol rêvé du père trouve de multiples passeurs et se prolonge ainsi à l'infini. Il n'est d'ailleurs pas anodin que la dernière étape de la chute, sur la dernière image de titre, nous montre Antonio à quelques centimètres du sol. Le personnage reste figé, en suspension dans les airs, comme si son vol ultime ne devait jamais prendre fin, grâce à la plume de son fils. Comme le dit Gaston Bachelard, "le vol onirique est la synthèse de la chute et de l'élévation $»^{16}$. Certes, le dernier vol d'Antonio n'est plus onirique mais il est bien la combinaison d'une chute réelle et d'une élévation éternelle grâce à l'écriture. 


\section{Conclusion}

Dans El arte de volar, la construction identitaire d'Antonio est mise en échec par la multiplication des obstacles. Les murs sont prison, séparation, entrave et matérialisent les difficultés à avancer lorsqu'on se construit au cœur d'une Espagne franquiste qui n'a de cesse d'empêcher l'individu d'exprimer ses désirs et ses idéaux. Le dépassement n'est possible que par le vol, un vol rêvé, car, pour reprendre les mots de Bachelard, « la vie onirique est d'autant plus pure qu'elle nous libère davantage de l'oppression des formes ${ }^{17}$. C'est pourquoi le père d'Altarriba «intenta volar a lo largo de toda su existencia, pero sus alas son cortas o la resistencia de la realidad es insalvable ${ }^{18}$. Face à ce déséquilibre des forces entre réalité et idéal, le vol est condamné à devenir chute, comme si le dépassement n'était possible que par le bas. Mais, si le franchissement des murs est impossible dans la vie, l'écriture a le pouvoir de les détruire, en donnant une lisibilité à tous les rêves de vol, au-delà des époques historiques. L'écriture serait alors le véritable « art de voler ».

\section{BIBLIOGRAPHIE}

Antonio ALTARRIBA, KIM, El arte de volar, Barcelona, Norma Editorial, 2018 ( $1^{\text {re }}$ édition 2009).

Gaston BACHELARD, L'air et les songes, Paris, Livre de Poche, 2001 (1 ${ }^{\text {re }}$ édition 1943).

Rafaela BRUMWELL, «La fenêtre : chronotope de la rêverie », Sociocriticism, vol. XV, 1, Université de Montpellier, 2000, p. 77-94.

Jean CHEVALIER, Alain GHEERBRANT, Dictionnaire des symboles, « Vol (air) », Paris, Robert Laffont, coll. Bouquins, 1982, p. 1027.

Juan Manuel DÍAZ DE GUEREÑU, Hacia un cómic de autor : a propósito de Arrugas y otras novelas gráficas, Bilbao, Universidad de Deusto, 2014.

Gilbert DURAND, Les structures anthropologiques de l'imaginaire, Paris, Dunod, 1992.

Lucía GONZÁLEZ, « El arte de volar, la memoria de la España triste hecha cómic » [on-line], El Mundo (11/04/2010) [consulté le 09/12/2021]

<URL https://www.elmundo.es/elmundo/2010/04/09/cultura/1270815946.html>.

Abel GRAU, «El arte de volar, crónica del choque de utopía y realidad en la España del siglo XX » [on-line], El País (16/11/2010) [consulté le 09/12/2021] <URL https://elpais.com/cultura/2010/11/16/actualidad/1289862004_850215.html>.

Benoît PEETERS, Lire la bande dessinée, Paris, Flammarion, 1998.

Alvaro PONS, « El arte de volar es una obra maestra » [on-line], El País (16/11/2010) [consulté le 09/12/2021]

<URL https://elpais.com/cultura/2010/11/16/actualidad/1289862010_850215.html>. 


\section{NOTES}

1. Les indications de pagination qui apparaissent dans cet article renvoient à l'édition suivante : Antonio Altarriba, Kim, El arte de volar, Barcelona, Norma Editorial, 2018.

2. Gilbert DURAND, Les structures anthropologiques de l'imaginaire, Paris, Dunod, 1992, p. 191.

3. Rafaela BRUMWELL, «La fenêtre : chronotope de la rêverie ", Sociocriticism, vol. XV, 1, Université de Montpellier, 2000, p. 78.

4. Id.

5. Gaston BACHELARD, L'air et les songes, Paris, Livre de Poche, 2001 (1 $1^{\text {re }}$ édition 1943), p. 55.

6. Ibid., p. 61.

7. Ibid., p. 79.

8. Ibid., p. 46.

9. Jean CHEVALIER, Alain GHEERBRANT, Dictionnaire des symboles, «Vol (air) », Paris, Robert Laffont, coll. Bouquins, 1982, p. 1027.

10. Gaston BACHELARD, op. cit., p. 30.

11. Alvaro PONS, «El arte de volar es una obra maestra » [on-line], El País (16/11/2010) [consulté le 09/12/2021]

<URL : https://elpais.com/cultura/2010/11/16/actualidad/1289862010_850215.html>.

12. Cité dans Juan Manuel DÍAZ DE GUEREÑU, Hacia un cómic de autor : a propósito de Arrugas y otras novelas gráficas, Bilbao, Universidad de Deusto, 2014, p. 190.

13. Paroles d'Antonio Altarriba, citées dans Lucía GONZÁLEZ, «El arte de volar, la memoria de la España triste hecha cómic » [on-line], El Mundo (11/04/2010) [consulté le 09/12/2021] <URL https://www.elmundo.es/elmundo/2010/04/09/cultura/1270815946.html>.

14. Abel GRAU, "El arte de volar, crónica del choque de utopía y realidad en la España del siglo XX » [on-line], El País (16/11/2010) [consulté le 09/12/2021] <URL https://elpais.com/cultura/ 2010/11/16/actualidad/1289862004_850215.html>.

15. Paroles d'Antonio Altarriba, citées dans Id.

16. Gaston BACHELARD, op. cit., p. 47.

17. Ibid., p. 36.

18. Abel GRAU, art. cit.

\section{RÉSUMÉS}

Dans El arte de volar de Antonio Altarriba et Kim, les murs et obstacles occupent une place essentielle, tant au niveau narratif qu'au niveau graphique. Le protagoniste apparaît sans cesse comme un homme empêché, enfermé entre les murs d'une réalité oppressante. Pourtant, loin de l'idée d'enfermement, le titre du roman graphique utilise le motif libérateur de l'envol. Ce que propose l'auteur est donc une dialectique entre le mur qui fait obstacle et l'envol qui permet le franchissement. Mais, si passer les murs suppose de voler, le vol est aussi généralement synonyme de chute, à l'image de la longue chute qu'est le suicide final du personnage qui structure la totalité du roman graphique. Mais cette chute est-elle le signe d'un échec ou peutelle être perçue comme l'envol ultime? Par l'hommage rendu à son père dans son roman graphique, Altarriba rend possible, grâce à l'écriture, le franchissement définitif des obstacles. 
Walls and obstacles play an essential role in Antonio Altarriba and Kim's El arte de volar, both from a narrative and graphical perspective. The protagonist is permanently portrayed as an uncomfortable man, detained inside the walls of an oppressing reality. Yet, far from the notion of imprisonment, the title of this graphic novel uses the liberating notion of flight. The author is therefore using a dialectic between the walls as obstacles, and the flight to overcome them. But if breaking through the walls requires flight, flying is also generally associated with falling, as illustrated by the final suicide of the character that provides the overall structure to the graphical novel. But is this fall an indication of failure, or can it be perceived as the ultimate flight? With this homage to his father, Altarriba uses writing as a way to overcome obstacles, once and for all.

INDEX

Mots-clés : Espagne, roman graphique, mémoire, obstacles, envol, écriture

Keywords : Spain, graphic novel, memory, obstacles, flight, writing

\section{AUTEUR}

\section{EMMANUELLE SOUVIGNET}

Université Jean Monnet - Saint-Etienne 